\title{
A Cheat-Proof Game Theoretic Demand Response Scheme for Smart Grids
}

\author{
Yan Chen, W. Sabrina Lin, Feng Han, Yu-Han Yang, Zoltan Safar, and K. J. Ray Liu \\ Department of Electrical and Computer Engineering, \\ University of Maryland, College Park, MD 20742, USA. \\ E-mail:\{yan, wylin, hanf, yhyang, zsafar, and kjrliu\}@umd.edu
}

\begin{abstract}
While demand response has achieved promising results on making the power grid more efficient and reliable, the additional dynamics and flexibility brought by demand response also increase the uncertainty and complexity of the centralized load forecast. In this paper, we propose a game theoretic demand response scheme that can transform the traditional centralized load prediction structure into a distributed load prediction system by the participation of customers. Moreover, since customers are generally rational and thus naturally selfish, they may cheat if cheating can improve their payoff. Therefore, enforcing truthtelling is crucial. We prove analytically and demonstrate with simulations that the proposed game theoretic scheme is cheatproof, i.e., all customers are motivated to report and consume their true optimal demands and any deviation will lead to a utility loss. We also prove theoretically that the proposed demand response scheme can lead to the solution that maximizes social welfare and is proportionally fair in terms of utility function. Moreover, we propose a simple dynamic pricing algorithm for the power substation to control the total demand of all customers to meet the target demand curve. Finally, simulations are shown to demonstrate the efficiency and effectiveness of the proposed game theoretic algorithm.
\end{abstract}

Index Terms-Smart grid, demand response, game theory, cheat-proof.

\section{INTRODUCTION}

Nowadays, traditional power grids are facing many challenges including load schedule uncertainties, low load factor and the incorporation of renewable energy systems. On the other hand, consumers are demanding better power quality and reliability, and the demand is arising due to the increase of new types of appliances. It is evidently needed to transform traditional electric network by using various smart devices, algorithms, and designs into a highly efficient and reliable power grid. A "Smart Grid" [1] is a future electricity delivery system based on new technologies, such as communication and market design. The goals are to enhance the reliability and quality of power supply, improve the efficiency of the power grid, and lower total energy consumption.

Demand response, one important feature of smart grid, is a mechanism that can motivate end-use customers to change their electricity usage from normal consumption patterns in response to the changes in the price of electricity over time [2]. Generally, there are mainly two different categories of demand response algorithms [2] [3]: incentive-based demand response and pricing-based demand response. In the incentive-based demand response, customers are given the incentive payments to reduce their consumptions in case of emergencies or when system reliability is jeopardized. For example, Chen et.al. [4] proposed to match the supply when the electricity supply is deficit based on supply function bidding. By shedding the load, customers can receive some revenue that is determined by the customers' supply functions. In pricing-based demand response, due to the use of dynamic pricing, customers will dynamically adjust their consumption according to the timevarying price to maximize their payoffs. In [5], the authors proposed a real-time pricing algorithm by maximizing the aggregate utility of all customers. With the presence of a realtime pricing tariff, Mohsenian-Rad and Leon-Garcia proposed a residential energy consumption scheduling algorithm by considering the trade-off between the electricity payment and the waiting time for the operation of each appliance [6].

The importance of demand response can go far beyond reducing the electricity bills of customers. It facilitates the demand management from the operational perspective through real-time pricing to balance the demand and supply in the electric power market [7] [8]. However, the additional dynamics and flexibility brought by demand response also increase the uncertainty and complexity of the centralized load forecast. This uncertainty will pose new challenges and make the difficult centralized load forecast problem even harder. In such a case, the forecast total demand from customers may not accurately reflect the future load. As a consequence, the power plant either over-generates or under-generates the power, which leads to low system efficiency and high risk of outage, respectively.

To overcome these challenges, we propose to use game theory to formulate the demand response problem. In this game, the players are the power substation and the customers, where the objective of the power substation is to dynamically adjust the total demand to meet a target demand curve and the objective of the customers is to maximize own their utilities. The main contributions are summarized as follows.

1) We propose a game theoretic demand response scheme that can transform the traditional centralized load prediction structure into a distributed load prediction system by the participation of customers. Such a transformation would revolutionize the difficult and complex centralized forecasting into easy demand report collection.

2) Since the proposed scheme is distributed and customers are naturally selfish, enforcing truth-telling is crucial. We prove that the proposed game theoretic scheme is cheatproof, i.e., all customers are motivated to report and consume their true optimal demands and any deviation will lead to a utility loss. 
3) We prove that the proposed demand response scheme can lead to the solution that is social welfare maximizing and proportionally fair in terms of the utility function.

4) To encourage participation, the demand response scheme should be simple since customers may not be willing to join the response scheme if the mechanism is complicated. The proposed demand response scheme is very simple. What customers need to do is to compute their own optimal demand for any given price and report the optimal demand to the power substation.

The rest of this paper is organized as follows. Section II introduces the system model and gain function. In Section III, we describe in details the proposed cheat-proof mechanism and the dynamic pricing of the power substation. Then, we show the simulation results to verify the proposed algorithm in Section IV. Finally, we draw conclusions in Section V.

\section{System Model and GAin FunCtion}

\section{A. System Model}

We consider a smart grid with $N$ customers that are served by one power substation. We assume that the power substation has a target total demand curve. The objective of the power substation is to control the total demand to meet the target demand curve by dynamically adjusting the price of the power. On the other hand, customers are intelligent and can make optimal decision based on the price of the power. The problem is to determine how the power substation should use dynamic pricing to control the total demand.

One possible approach is the centralized approach where the power substation predicts the total demand by estimating the behavior of the customers using power consumption history. However, this kind of centralized approach has several drawbacks. Firstly, the computational complexity at the power substation is very high since the power substation needs to estimate the behaviors of all customers and predict their power consumption. Secondly, the prediction accuracy of the total demand consumption may not be high due to the complex behavior of customers. Thirdly, due to the inaccurate prediction, the power substation may over-generate or under-generate power which leads to power inefficiency or power outage.

To overcome these drawbacks, we propose to use game theory to formulate the demand response problem. In this game, the players are the power substation and the $N$ customers, where the objective of the power substation is to use realtime pricing to dynamically adjust the total demand to meet a certain target demand and the objective of the customers is to dynamically choose the optimal demand to maximize their own utilities. With such a game theoretic formulation, we do not require the power substation to centrally estimate the power consumption of each customer. Instead, we ask the customers to report their demands to the power substation. Since customers are rational and thus naturally selfish, they tend to over-claim what they may need and will not truly report their optimal demand if cheating can improve their payoffs. To enforce customers to report their true optimal demands, we propose a cheat-proof mechanism by carefully designing the penalty function for deviation.

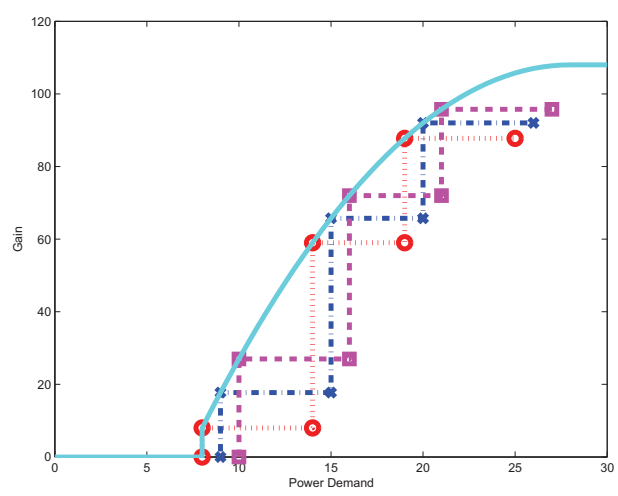

Fig. 1. The illustration of the gain function.

\section{B. Gain function of the Customer}

Generally, by consuming a certain amount of power, a customer experiences a certain gain which reflects the level of satisfaction. For example, when reading under a bad lighting condition, a customer will feel happy by turning on a light, and such a happiness can be characterized by a certain gain. We assume that each customer $i$ has a minimal power demand $d_{i}^{\text {min }}$ to operate some essential appliances such as refrigerator and air conditioner. If the demand is smaller than $d_{i}^{\text {min }}$, the customer will feel very uncomfortable, so the gain is zero. Once the power demand reaches $d_{i}^{\text {min }}$, the customer experiences a gain $g_{i}$. After satisfying the minimal demand constraint, the gain will increase by a certain value if an additional appliance is turned on. Since customers are intelligent and rational, they will always first turn on the appliance that can bring the largest increase of their level of satisfaction. For example, a customer in a dark room will turn on the light first while the customer who feels hot will turn on the fan first. With such an intuition, the gain function should be an increasing step function with the step size decreasing over the demand for all demand that is larger than the minimal demand constraint, e.g. the red dot curve in Figure 1. Since the gain function characterizes the level of satisfaction of the customer, it can be different under different climate conditions and different moods of the customer. For example, the pink dash curve and blue dot-dash curve in Figure 1 can be the gain functions under two different conditions. To better understand the behavior of the customers, we assume that the gain function measures the average level of satisfaction under different conditions such as climate and mood conditions.

From the above discussion, we can see that the gain should be zero for any demand smaller than the minimal demand constraint. For the demand larger than the minimal demand constraint, the gain function should be a monotonically increasing concave function with the level of satisfaction gradually saturating. After the demand reaching the maximal demand constraint which can be the total power demand for operating all appliances, the gain function should remain constant. There are many possible gain functions. In this paper, we use a modified quadratic gain function $G_{i}(d)$ defined in (1) and illustrated as the cyan curve in Figure 1. A similar 
gain function was used in [5].

$$
G_{i}(d)= \begin{cases}0, & \text { if } 0 \leq d<d_{i}^{\text {min }} ; \\ -\frac{\alpha_{i}}{2}\left(d-d_{i}^{\text {min }}\right)^{2} & \\ +w_{i}\left(d-d_{i}^{m i n}\right)+g_{i}, & \text { elseif } d \leq \frac{w_{i}}{\alpha_{i}}+d_{i}^{\text {min }} ; \\ \frac{w_{i}^{2}}{2 \alpha_{i}}+g_{i}, & \text { else, }\end{cases}
$$

where $d_{i}^{\text {min }}$ is the minimal power demand, $g_{i}$ is the gain of the minimal power demand, $\alpha_{i}$ is the parameter controlling the speed of increase of the quadratic function which can be timevarying, and $w_{i}$ is the declivity of the parabola at $d=d_{i}^{\text {min }}$ which is fixed over time.

\section{Demand RESPONSE GAMES}

To motivate customers to report their true optimal demands to power substation, we propose a cheat-proof demand response scheme as follows. At the beginning of time slot $t$, the power substation announces a reference price $p^{r}$ to all customers. After receiving the reference price, each customer computes the optimal demand $d_{i}^{\star}$ by maximizing the intermediate utility function as follows,

$$
\begin{gathered}
d_{i}^{\star}=\arg \max _{d} \lambda G_{i}(d)-p^{r} d \\
= \begin{cases}\frac{w_{i}-p^{r} / \lambda}{\alpha_{i}}+d_{i}^{\text {min }}, & \text { if } \frac{\left(\lambda w_{i}-p^{r}\right)^{2}}{2 \lambda \alpha_{i}}+\lambda g_{i} \geq p^{r} d_{i}^{\text {min }} \\
d_{i}^{\text {min }}, & \text { and } w_{i} \geq p^{r} / \lambda, \\
0, & \text { if } w_{i}<p^{r} / \lambda \text { and } \lambda g_{i} \geq p^{r} d_{i}^{\text {min }}, \\
\text { else, },\end{cases}
\end{gathered}
$$

where $\lambda$ is a parameter balancing gain $G_{i}(d)$ and cost $p^{r} d$.

Due to selfish nature, customers may not report their true optimal demand $d_{i}^{\star}$. Let us assume that customer $i$ reports $\hat{d}_{i}$ to the power substation. After receiving all the demands from customers, the power substation announces to each customer $i$ the price $p_{i}$ and the cost function $C_{i}\left(p_{i}, d_{i}, \hat{d}_{i}\right)$ as follows

$$
\begin{gathered}
p_{i}=p^{r}+\frac{m}{\hat{d}_{i}} \\
C_{i}\left(p_{i}, d_{i}, \hat{d}_{i}\right)= \begin{cases}p_{i} \hat{d}_{i}, & \text { if } d_{i} \leq \hat{d}_{i} ; \\
p_{i} \hat{d}_{i}+\lambda \Delta\left(d_{i}-\hat{d}_{i}\right)+\lambda \rho, & \text { if } d_{i}>\hat{d}_{i} .\end{cases}
\end{gathered}
$$

where $m$ is a constant which can be treated as the maintenance fee, $\Delta \geq \max \left\{w_{1}, \ldots, w_{N}\right\}$ and $\rho \geq \max \left\{g_{1}, \ldots, g_{N}\right\}$ are two fixed parameters. From (4), we can see that customers are punished for both over-reporting and under-reporting. For over-reporting, customers will be charged for what they reported. For under-reporting, customers will receive a penalty that is linear in the deviation $d_{i}-\hat{d}_{i}$.

Finally, according to the price $p_{i}$, each customer consumes power $d_{i}$ and pays $C_{i}\left(p_{i}, d_{i}, \hat{d}_{i}\right)$, and the utility function of customer $i$ can be computed as

$$
U_{i}\left(d_{i}, \hat{d}_{i}\right)=\lambda G_{i}\left(d_{i}\right)-C_{i}\left(p_{i}, d_{i}, \hat{d}_{i}\right)
$$

\section{A. Cheat-Proof Property}

In this subsection, we prove that the proposed mechanism is cheat-proof, which means that all customers are motivated to report and consume the optimal demands, i.e., their utility is maximized when $d_{i}=\hat{d}_{i}=d_{i}^{\star}$, and any deviation will lead to a utility loss.
Lemma 1: After reporting $\hat{d}_{i}$, the best strategy of customer $i$ at the consumption stage is to consume $\hat{d}_{i}$, i.e., $U_{i}\left(d_{i}, \hat{d}_{i}\right) \leq$ $U_{i}\left(\hat{d}_{i}, \hat{d}_{i}\right), \forall d_{i}$, with equality if and only if $d_{i}=\hat{d}_{i}$.

Proof: Due to page limitation, we show the proof in the supplementary information [9].

Theorem 1: The proposed mechanism is cheat-proof.

Proof: To prove Theorem 1, we need to show that the utility of the customer is maximized at $d_{i}=\hat{d}_{i}=d_{i}^{\star}$.

According to (2), (3), (4), (5) and Lemma 1, we have

$$
\begin{aligned}
U_{i}\left(d_{i}, \hat{d}_{i}\right) & \leq U_{i}\left(\hat{d}_{i}, \hat{d}_{i}\right)=\lambda G_{i}\left(\hat{d}_{i}\right)-C_{i}\left(p_{i}, \hat{d}_{i}, \hat{d}_{i}\right) \\
& =\lambda G_{i}\left(\hat{d}_{i}\right)-p_{i} \hat{d}_{i}=\lambda G_{i}\left(\hat{d}_{i}\right)-p^{r} \hat{d}_{i}-m \\
& \leq \lambda G_{i}\left(d_{i}^{\star}\right)-p^{r} d_{i}^{\star}-m .
\end{aligned}
$$

The equality in (6) holds if and only if $d_{i}=\hat{d}_{i}=d_{i}^{\star}$. Therefore, all customers will report and consume the optimal demand, and any deviation will lead to a utility loss, i.e., the proposed mechanism is cheat-proof.

\section{B. Maximizing Social Welfare}

In this subsection, we prove that the proposed mechanism leads to an equilibrium that maximizes the social welfare, which is the sum of the customers' utilities and the revenue of the substation.

Theorem 2: The proposed mechanism maximizes the social welfare, i.e., $\left(d_{1}^{\star}, d_{2}^{\star}, \ldots, d_{N}^{\star}\right)$ is the solution to the following optimization problem

$$
\max _{d_{i}, \forall i} \sum_{i=1}^{N} G_{i}\left(d_{i}\right) \text { s.t. } \sum_{i=1}^{N} d_{i} \leq d^{\text {total }}
$$

with $d^{\text {total }}=\sum_{i=1}^{N} d_{i}^{\star}$.

Proof: Due to page limitation, we show the proof in the supplementary information [9].

\section{Proportionally Fair Solution}

In this subsection, we prove that the proposed mechanism leads to the solution that is proportionally fair in utility.

Theorem 3: The proposed mechanism leads to the solution that is proportionally fair in utility, i.e., $\left(d_{1}^{\star}, d_{2}^{\star}, \ldots, d_{N}^{\star}\right)$ is the solution to the following optimization problem

$$
\max _{d_{i}, \forall i} \prod_{i=1}^{N}\left(G_{i}\left(d_{i}\right)-p^{r} d_{i}\right) .
$$

Proof: Due to page limitation, we show the proof in the supplementary information [9].

\section{Dynamic Pricing of the Power Substation}

From the above discussion, we can see that the proposed mechanism is cheat-proof, due to which all rational customers are motivated to report and consume the optimal demand. According to (2), the average power consumption of the customers at time slot $t$ can be computed as follows

$$
D^{\text {average }}(t)=\frac{1}{N} \sum_{i=1}^{N}\left(\frac{w_{i}-p^{r}(t) / \lambda}{\alpha_{i}(t)}+d_{i}^{\text {min }}\right) .
$$

Here, we exclude the inactive customers whose optimal demand is zero and simply assume $N$ is the total number 
of active customers. Moreover, we assume that the parameters $w_{i}$ and $d_{i}^{\text {min }}$ are time-invariant parameters while the parameter $\alpha_{i}(t)$ is a time-variant parameter.

As discussed in Section II-B, $\alpha_{i}(t)$ is the parameter controlling the speed of increase of the quadratic gain function. According to (9), if $\frac{1}{\alpha_{i}(t)}$ is modeled as a Gaussian distribution with mean $\mu_{\alpha}(t)$ and constant variance $\sigma_{\alpha}^{2}$, then, with the law of large numbers, the average power consumption of all customers can be approximated as

$$
\begin{aligned}
D^{\text {average }}(t) & \approx \mu_{\alpha}(t)\left(\frac{1}{N} \sum_{i=1}^{N} w_{i}-p^{r}(t) / \lambda\right)+\frac{1}{N} \sum_{i=1}^{N} d_{i}^{\text {min }}, \\
& =\mu_{\alpha}(t)\left(W-p^{r}(t) / \lambda\right)+Q,
\end{aligned}
$$

where $W=\frac{1}{N} \sum_{i=1}^{N} w_{i}$ and $Q=\frac{1}{N} \sum_{i=1}^{N} d_{i}^{\text {min }}$.

From (10), we can see that there is a direct relationship between $p^{r}(t)$ and $D^{\text {average }}(t)$. Therefore, by dynamically adjusting $p^{r}(t)$, we can control the average demand $D^{\text {average }}(t)$ to meet the target average demand $D^{\text {target }}(t)$ as follows

$$
p^{r}(t)=\lambda\left[W-\frac{D^{\text {target }}(t)-Q}{\mu_{\alpha}(t)}\right] .
$$

Since $W$ and $Q$ are two constants, we assume they are known or can be well estimated by the power substation. The $\mu_{\alpha}(t)$ is the mean of the parameter $\frac{1}{\alpha_{i}(t)}$ controlling the increasing speed of the gain function, which means that $\mu_{\alpha}(t)$ should be reasonably smooth over $t$. Therefore, we use AutoRegressive (AR) process to model $\mu_{\alpha}(t)$, i.e., $\mu_{\alpha}(t)$ can be represented as a linear combination of $\mu_{\alpha}(t-1), \ldots, \mu_{\alpha}(t-l)$ with an additive white Gaussian noise,

$$
\mu_{\alpha}(t)=\gamma_{1} \mu_{\alpha}(t-1)+\ldots+\gamma_{m} \mu_{\alpha}(t-l)+n_{\mu_{\alpha}}(t) .
$$

According to the discussions above, the dynamic pricing algorithm of the power substation can be summarized as follows. At time slot $t$, the power substation announces a reference price $p^{r}(t)$. Based on $p^{r}(t)$, all customers report their optimal demand. After receiving all demands from customers, the power substation computes $\mu_{\alpha}(t)$ using

$$
\mu_{\alpha}(t)=\frac{D^{\text {average }}(t)-Q}{W-p^{r}(t) / \lambda},
$$

and estimates $\mu_{\alpha}(t+1)$ using

$$
\hat{\mu}_{\alpha}(t+1)=\gamma_{1} \mu_{\alpha}(t)+\ldots+\gamma_{l} \mu_{\alpha}(t-l+1) .
$$

Finally, based on the estimated $\mu_{\alpha}(t+1)$, the power substation computes $p^{r}(t+1)$ using

$$
p^{r}(t+1)=\lambda\left[W-\frac{D^{\text {target }}(t+1)-Q}{\hat{\mu}_{\alpha}(t+1)}\right] .
$$

\section{Simulation Results}

In this section, we show with simulations that our proposed game theoretic scheme is cheat-proof and can effectively control the power consumption. We consider a system with one power substation and $N=1000$ customers. The variables in the gain function (1), $1 / \alpha_{i}, w_{i}$, and $d_{i}^{\text {min }}$, are assumed to be Gaussian, i.e., $1 / \alpha_{i}(t) \sim N\left(\mu_{\alpha}(t), \sigma_{\mu}\right), w_{i} \sim N\left(\mu_{w}, \sigma_{w}\right)$, and $d_{i}^{\min } \sim N\left(\mu_{d}, \sigma_{d}\right)$. The parameters used in our model

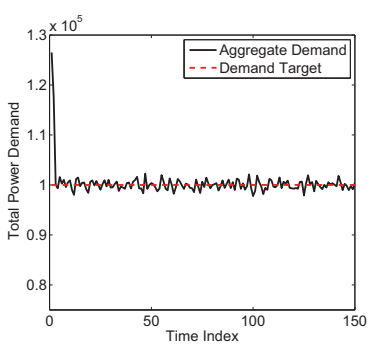

(a)

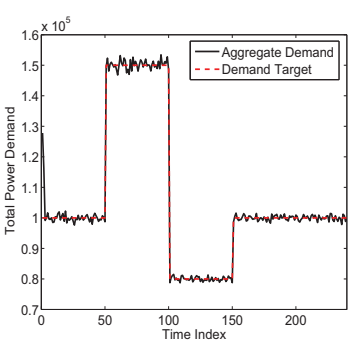

(b)
Fig. 4. The demand controlling performance of the proposed scheme: (a) constant target demand; (b) time-varying target demand.

are set as: $\mu_{w}=150, \sigma_{w}=25, \mu_{d}=5, \sigma_{d}=1, \sigma_{\mu}=0.2$, $\lambda=0.02, g_{i}=1000$ and $m=5$. We use $\operatorname{AR}(2)$ process to model $\mu_{\alpha}(t)$ with AR coefficients $\gamma_{1}=0.6$ and $\gamma_{2}=0.4$.

In the first simulation, we verify the cheat-proof performance of the proposed scheme. Figure 2 (a) shows the utility versus the real power consumption $d_{i}$ by assuming the reported demand is equal to the real consumption, i.e., $d_{i}=\hat{d}_{i}$. We can see that the maximal utility is achieved when $d_{i}=\hat{d}_{i}=d_{i}^{\star}=$ 73 , which means that if the customer will consume the same amount of power as what he reported, the best strategy for the customer is to report the optimal demand $\hat{d}_{i}=d_{i}^{\star}$. In Figure 2 (b), we show the utility versus $d_{i}$ by assuming $\hat{d}_{i}=d_{i}^{\star}$. We can see that the utility is maximized when $d_{i}=\hat{d}_{i}=d_{i}^{\star}=73$. Therefore, after reporting $\hat{d}_{i}$, customer has no incentive to consume $d_{i} \neq \hat{d}_{i}$ due to the punishment for both consuming more and less power in (4). Then, in Figure 2 (c), we shown the utility versus $\hat{d}_{i}$ by assuming $d_{i}=d_{i}^{\star}$. We can again see that only when $\hat{d}_{i}=d_{i}=d_{i}^{\star}$, the utility is maximized, which means that customers will report the true optimal demand to achieve maximal utility. The customer's utility versus $\left(\hat{d}_{i}, d_{i}\right)$ is shown in Figure $2(\mathrm{~d})$. We can see that any $\left(\hat{d}_{i}, d_{i}\right)$ other than $\left(d_{i}^{\star}, d_{i}^{\star}\right)$ leads to a loss of utility. Hence, customers have no incentive report or consume other than $d_{i}^{\star}$, and our proposed game theoretic scheme is cheat-proof.

From the above discussions, we can see that the proposed scheme is cheat-proof. Such a cheat-proof property is mainly because of the use of the punishment term in (4). Without the punishment, customers may not report and consume the optimal demand. In Figure 3, we show the customer's utility versus $\left(\hat{d}_{i}, d_{i}\right)$ by assuming no punishment term in the utility function, i.e., the cost function is defined as $p^{r} d_{i}$. As shown in Figure 3, we can see that for any given $\hat{d}_{i}$, the utility is maximized when $d_{i}=d_{i}^{\star}$. However, the utility function is independent from $\hat{d}_{i}$, i.e., the utility function is constant in terms of $\hat{d}_{i}$. In such a case, customers have no incentive to report true optimal demand. Therefore, it is very important for us to involve the punishment term in the cost function.

We then evaluate the performance of the proposed dynamic pricing algorithm in Figure 4. We can see from Figure 4 (a) that with the proposed dynamic pricing algorithm, the total demand from customers quickly converges to the target total demand with the difference between the total demand and the target demand less than $2 \%$ of the target demand. From Figure 4 (b), we can see that the proposed algorithm can quickly 


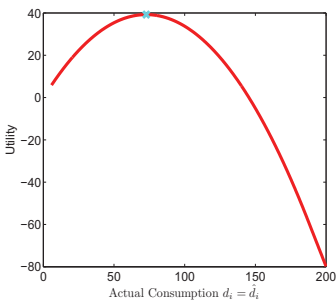

(a)

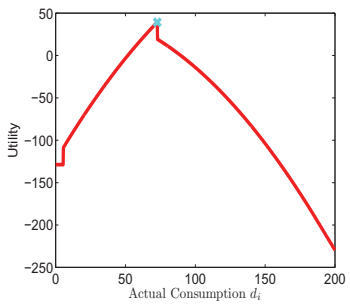

(b)

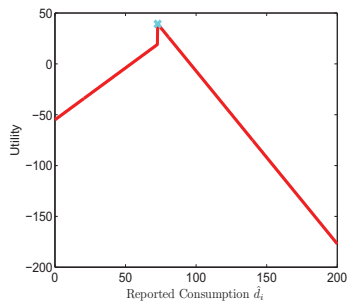

(c)

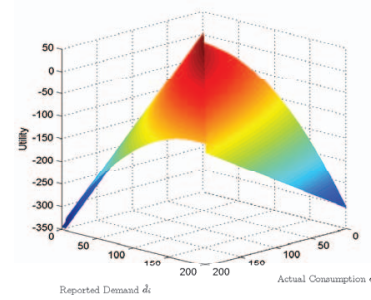

(d)

Fig. 2. The cheat-proof performance of the proposed scheme when the optimal demand $d_{i}^{\star}=73$ and the reference price $p^{r}=1.7$ : (a) the utility versus the $d_{i}$ when $\hat{d}_{i}=d_{i}$; (b) the utility versus $d_{i}$ when $\hat{d}_{i}=d_{i}^{\star}=73$; (c) the utility versus $\hat{d}_{i}$ when $\hat{d}_{i}=d_{i}^{\star}=73$; (d) the utility versus $\hat{d}_{i}$ and $d_{i}$.

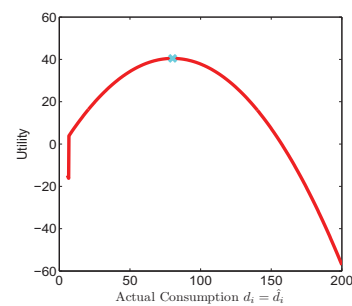

(a)

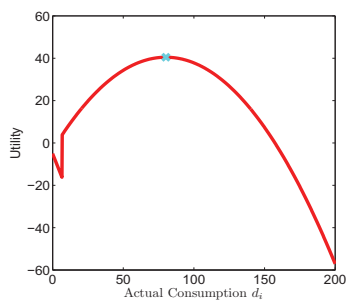

(b)

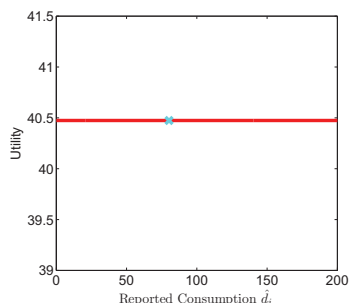

(c)

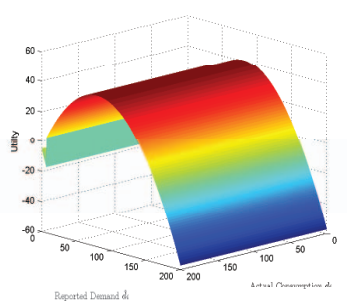

(d)

Fig. 3. The non-cheat-proof performance of the scheme without punishment in the utility function: (a) the utility versus $d_{i}$ when $\hat{d}_{i}=d_{i}$; (b) the utility versus $d_{i}$ when $\hat{d}_{i}=d_{i}^{\star}=80$; (c) the utility versus $\hat{d}_{i}$ when $\hat{d}_{i}=d_{i}^{\star}=80$; (d) the utility versus $\hat{d}_{i}$ and $d_{i}$.

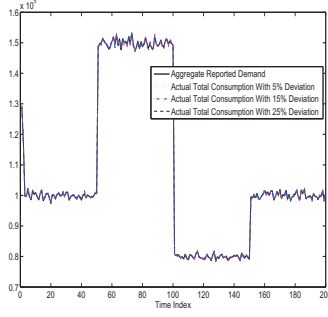

(a)

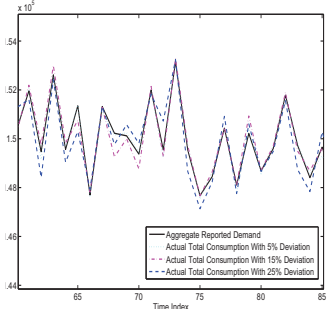

(b)
Fig. 5. (a) The robustness performance against real consumption deviation constant target demand; (b) zoom-in figure of (a).

respond to the change of target demand through adjusting the price accordingly, which fully demonstrate the efficiency and effectiveness of the proposed dynamic pricing algorithm.

In Figure 5, we evaluate the effect of customers' deviation on the total demand. We assume that there is a portion of customers deviating from their reported demands. The customer who deviates will consume $(1+\epsilon)$ times of the reported demand, i.e., $d_{i}=(1+\epsilon) \hat{d}_{i}$, where $\epsilon$ satisfies Gaussian distribution with zero mean and 0.2 variance. From Figure 5 , we can see that even with $25 \%$ of customers deviating from the reported demands, the actual total consumption is still close to the aggregate reported demand. Therefore, the proposed scheme is very robust to the customers' deviation.

\section{Conclusions}

In this paper, we have proposed a game theoretic demand response scheme that can transform the difficult and complex centralized demand forecasting problem into an easy demand reporting mechanism. Such a game theoretic scheme is shown to be cheat-proof which can enforce selfish customers to report and consume their optimal demands. Moreover, the proposed scheme leads to the desired solution from both system designer's and customers' perspective since the solution is not only social welfare maximizing but also proportionally fair in utility. Furthermore, we have proposed a simple dynamic pricing algorithm to effectively control the total demand to meet a target demand curve. With the proposed algorithm, the power substation can obtain an accurate estimate of the real consumption ahead of time and generate the corresponding amount of power, and thus greatly improve the system efficiency and reduce the risk of outage.

\section{REFERENCES}

[1] The Smart Grid: An Introduction. U.S. Department of Energy, 2005.

[2] U. D. of Energy, "Benefits of demand response in electricity markets and recommendations for achieving them," in Report to the United States Congress, February 2006.

[3] M. H. Albadi and E. F. El-Saadany, "A summary of demand response in electricity markets," Electric Power Systems Research, vol. 78, no. 11, pp. 1989-1996, 2008.

[4] L. Chen, N. Li, S. Low, and J. Doyle, "Two market models for demand response in power networks," in Smart Grid Communications (SmartGridComm), 2010 First IEEE International Conference on, pp. 397 -402, oct. 2010.

[5] P. Samadi, A. Mohsenian-Rad, R. Schober, V. Wong, and J. Jatskevich, "Optimal real-time pricing algorithm based on utility maximization for smart grid," in Smart Grid Communications (SmartGridComm), 2010 First IEEE International Conference on, pp. 415 -420, oct. 2010.

[6] A.-H. Mohsenian-Rad and A. Leon-Garcia, "Optimal residential load control with price prediction in real-time electricity pricing environments," IEEE Transactions on Smart Grid, vol. 1, no. 2, pp. 120-133, 2010.

[7] G. Barbose, C. Goldman, and C. Neenan, "A survey of utility experience with real time pricing," Lawrence Berkeley National Laboratory Report No. LBNL-54238, 2004.

[8] J. Zhang, J. D. Fuller, and S. Elhedhli, "A stochastic programming model for a day-ahead electricity market with real-time reserve shortage pricing," IEEE Transactions on Power Systems, vol. 25, pp. 703-713, 2010.

[9] Supplementary information: http://www.ece.umd.edu/ yan/SI.pdf. 